\title{
Non-obtuse Remeshing with Centroidal Voronoi Tessellation
}

\author{
Dong-Ming Yan, Peter Wonka
}

\begin{abstract}
We present a novel remeshing algorithm that avoids triangles with small and triangles with large (obtuse) angles. Our solution is based on an extension to Centroidal Voronoi Tesselation (CVT). We augment the original CVT formulation by a penalty term that penalizes short Voronoi edges, while the CVT term helps to avoid small angles. Our results show significant improvements of the remeshing quality over the state of the art.
\end{abstract}

\section{INTRODUCTION}

- D.-M. Yan is with the King Abdullah University of Science and Technology, Thuwal 23955-6900, Saudi Arabia, and the National Labord68 tory of Pattern Recognition, Institute of Automation, Chinese Academb9 of Sciences, Beijing 100190, China.E-mail: yandongming@gmail.com.

- P. Wonka is with the King Abdullah University of Science and Technology, Thuwal 23955-6900, Saudi Arabia, and Arizona State 1 University, Tempe, AZ 85287-8809, USA. E-mail: pwonka@gmail.com² Polygonal geometry processing has contributed many useful techniques to generate, manipulate, and manuacture three-dimensional models. Examples are mesh moothing, mesh deformation, parameterization, sur $_{\overline{3} 7}$ ace approximation, and mesh segmentation [1]. $A_{8}$ significant number of these techniques is based on $_{39}$ solving PDEs with the Laplacian matrix being in $\overline{4}_{\overline{4}}$ volved in the computation. Therefore, many recent remeshing techniques focus on eliminating triangle with small angles. The condition number of the Lapla $a_{\overline{4} 3}$ cian matrix is directly affected by small angles and even a single bad triangle can have a big impact on the computation [2]. Somewhat surprisingly, there is very little work on triangular remeshing for avoiding large (obtuse) angles, even though obtuse angles have $a_{8}$ similar bad impact on the computation. For example the correctness of the fast marching method highly $y_{0}$ depends on the mesh quality. The obtuse angles in the mesh might violate the order of the node processing In order to setup the Laplacian matrix, the defacto ${ }_{3}$ standard is to use the cotan Laplacian [3]. The cotan ${ }_{4}$ Laplacian is guaranteed to yield positive values if no obtuse triangles are present. Otherwise, negative ${ }_{6}$ values might occur requiring special treatment $i_{37}$ many common algorithms, e.g., [4].

In this paper, we present a novel remeshing tech nique that avoids both small angles $\left(<30^{\circ}\right)$ and obtuse angles $\left(\geq 90^{\circ}\right)$. Our main idea is to add an additional penalty term to improve state-of-the-art CVT-based remeshing. To prevent obtuse angles, $\mathrm{W}_{\mathrm{E}_{3}}$ penalize short edges in the dual mesh, the Voronod diagram. Our results show that we can produce ver ${ }_{65}$ good remeshing results on a wide range of models and significantly improve upon the state of the art.

\section{Related Work}

There are many different techniques to remesh a surface, e.g., mesh simplification [5], mesh optimization with local operations [6], the active-front based method [7], Delaunay insertion algorithms [8] and variational approaches. The remeshing techniques can also be classified as isotropic or anisotropic [9], [10]. In this paper we focus on isotropic remeshing. A complete survey of all the remeshing techniques is beyond the scope of this paper. We will concentrate on the approaches for isotropic remeshing that are based on Centroidal Voronoi tessellation. We refer the reader to a survey paper [11] for more details about remeshing and a text book [12] for the fundamentals of the Delaunay triangulation and the Voronoi diagram.

Centroidal Voronoi tessellation [13] has been used as starting point for many remeshing techniques. The difference between the available methods is how the Voronoi diagram on surfaces is approximated. The parameterization based approaches [14], [15], [16], [17] first parameterize the 3D mesh locally or globally, and then perform Lloyd iterations [18] in the 2D parameter domain and finally map the 2D samples back to the original 3D surface. Rong et al. [19] propose to use the hyperbolic space as the parameter domain. Parametrization-based approaches are fast and efficient, but suffer from the distortion introduced by the parameterization. Instead of parametrizing the 3D surface, Valette et al. [20] directly cluster triangles of a 3D mesh and compute an approximated CVT on the mesh surface.

Another way of computing CVT on surfaces is to use the centroidal Geodesic Voronoi Diagram (GVD) [21], [22], [23]. Although the centroidal GVD can sometimes generate good quality meshes, there is no direct link between the meshing quality and the centroidal GVD, since the quality of the remeshed surfaces are measured with the Euclidean metric. 


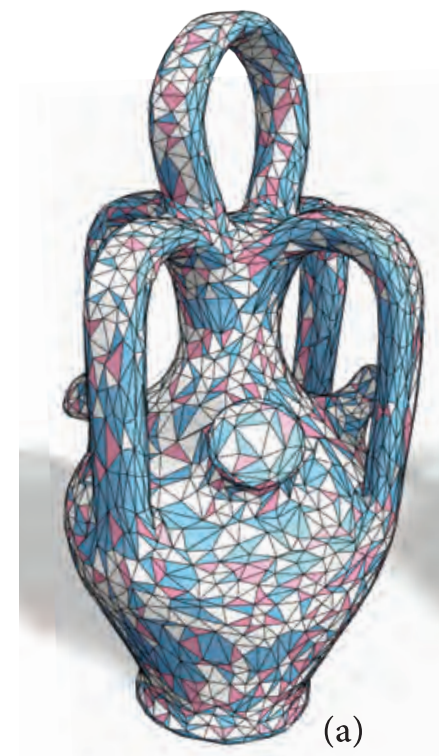

(a)

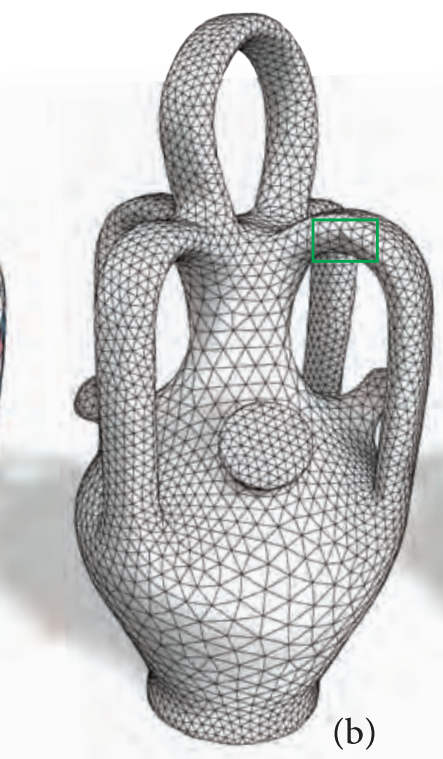

(b)

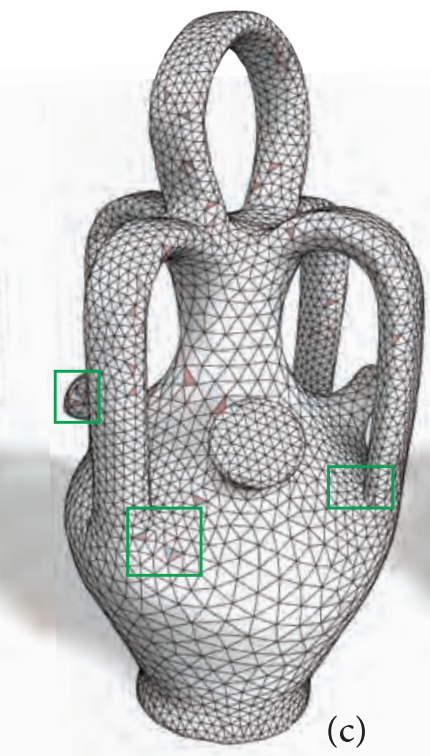

(c)

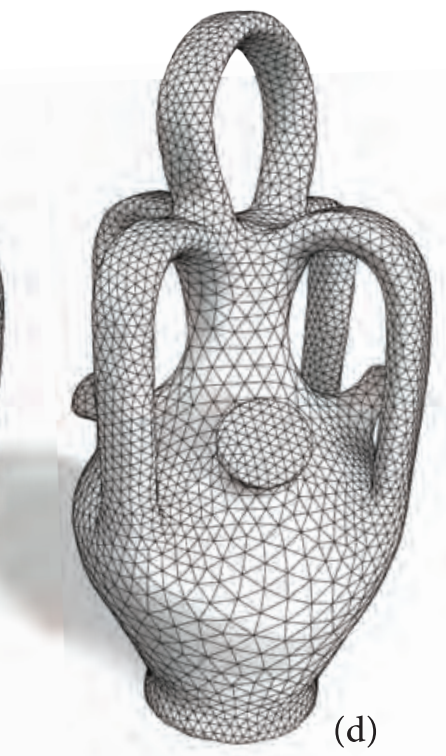

(d)

Fig. 1. Non-obtuse remeshing of the Botijo model with $5 \mathrm{~K}$ vertices and $10 \mathrm{~K}$ triangles. The pink triangles are obtuse and the blue triangles have small angles, i.e., less than 30 degrees. (a) Input mesh with $6 k$ triangles; (b) result of CVT after 300 iterations (8 obtuse triangles); (c) result of feature sensitive CVT after 200 iterations (231 obtuse triangles and 6 triangles with small angles); and (d) our non-obtuse remeshing result with 100 iterations. Our result has no obtuse and no small angles.

While future work might generate good remeshing reor sults using geodesic distances, currently the methodis based on Euclidean distances create superior resultsas Moreover, we are not aware of any existing worka that uses a geodesic metric to generate non-obtuseo remeshings on surfaces.

To build a state-of-the-art remeshing technique, we believe it is the best idea to compute the exact $R e^{13}$ stricted Voronoi Diagram (RVD) [24] and not to rely4 on approximations. For the efficient optimization of the CVT energy, Liu et al. [25] propose to use $\mathrm{a}^{6}$ Newton-like solver, e.g., L-BFGS. Based on these two techniques, an efficient remeshing framework can bes developed, which overcomes the drawbacks of pre ${ }^{19}$ vious parameterization-based and discrete clustering 20 based methods. Lévy and Bonneel [26] propose ther Voronoi parallel linear enumeration that uses $A p^{22}$ proximate Nearest Neighbor (ANN) search instead of 3 Delaunay triangulation to improve the efficiency of the RVD computation. Lévy [27] further improves $2_{4}$ the robustness of RVD computation using a combi $\dot{i}_{25}$ nation of arithmetic filters, expansion arithmetics and $\mathbb{1}_{6}$ symbolic perturbation. More recently, Yan et al. [28] propose the localized RVD that enables remeshing with a low number of polygons. The RVD framework $x_{9}$ is also used for blue-noise sampling and remeshing op surfaces [29], [30], [31], polynomial surface fitting [32] and the modeling of minimal surfaces [33].

A related concept is the "well-centered" trianguss lation where the circumscribed centers have to bet located inside the corresponding triangles. Note that $5_{5}$ "well-centered" and "nonobtuse" triangle meshes aneo equivalent if vertex weights are not considered. Sieger et al. [34] propose to remove the short Voronoi edges by minimizing the summed squared distances between inscribed and circumscribed centers of each triangle in order to improve the condition number of the Laplacian matrix. Vanderzee et al. [35] study the theoretical aspect of well centered triangulations in Euclidean space. Sun et al. [36] proposed to reduce the number of obtuse triangles in anisotropic remeshing by replacing the Euclidean metric with a hexagonal Minkowski metric. In the context of weighted triangulation optimization, Mullen et al. [37] propose to use the power diagram and regular triangulation to compute well-centered primal/dual meshes. More recently, de Goes et al. [4] improve primal/dual meshes using weighted triangulation. However, both methods cannot guarantee the non-obtuse property of the optimized meshes.

However, none of the methods discussed above is able to generate a mesh without obtuse triangles, which is very important in mesh processing, e.g., FEM simulation [38]. Non-obtuse meshing has been investigated in the 2D plane [39], [40], [41], [42], but this work does not directly apply to our 3D remeshing problem. The only approach for isotropic surface remeshing we are aware of is [43], which uses the quadric-based mesh decimation algorithm to generate non-obtuse meshes. Unfortunately, their method cannot prevent small angles. In this paper, we will address the problem of generating high quality meshes, without both small angles and obtuse angles. 


\section{Remeshing Framework}

Considering the huge amount of literature in remeshing, it becomes a daunting task to determine the stateof-the-art. In this section, we propose a combination of multiple published techniques that we believe will give the best results among the currently published methods and we will give some explanation why obtuse triangles cannot be avoided. The building blocks we propose to use are: 1) CVT 2) The exact Restricted Voronoi Diagram (RVD) 3) The Feature Sensitive CVT extension and 4) Optimization based on L-BFGS.

The input is a mesh surface $\mathcal{M}=\{F, V\}$, and a set of samples $\mathbf{X}=\left\{\mathbf{x}_{i}\right\}_{i=1}^{n}$ generated on $\mathcal{M}$. $\mathcal{M}$ is a 2-manifold triangular mesh consisting of a set of vertices $V$ and faces $F$. The goal is to compute the CVT of the samples on the surface and to extract $\mathrm{a}^{7}$ primal mesh from the optimized samples that satisfiess certain properties.

\subsection{Centroidal Voronoi tessellation}

Centroidal Voronoi tessellation of $n$ distinct points ( $\mathrm{O}^{2}$ seeds) $\mathbf{X}=\left\{\mathbf{x}_{i}\right\}_{i=1}^{n}$ in $R^{3}$ is a special kind of Vorondis tessellation that minimizes the following energy fun $\mathrm{C}^{84}$ tion [13]:

$$
F_{C V T}(\mathbf{X})=\sum_{i=1}^{n} \int_{\Omega_{i}} \rho(\mathbf{x})\left\|\mathbf{x}-\mathbf{x}_{i}\right\|^{2} \mathrm{~d} \sigma,
$$

where $\Omega_{i}=\left\{\mathbf{x} \in R^{3} \mid\left\|\mathbf{x}-\mathbf{x}_{i}\right\| \leq\left\|\mathbf{x}-\mathbf{x}_{j}\right\|, \forall j \neq i\right\}$ is the Voronoi cell of point $\mathbf{x}_{i}$, and $\rho(\mathbf{x}) \geq 0$ is a densitys function defined over the domain.

\subsection{Restricted Voronoi diagram}

To compute the CVT on mesh surfaces, we have to restrict the Voronoi diagram on surfaces, called 3 restricted Voronoi diagram (RVD) [44]. It is defined a $\mathrm{s}_{4}$ the intersection of the 3D Voronoi diagram $\Omega=\left\{\Omega_{i 165}\right.$ and the mesh surface, i.e., $\Omega_{\mid \mathcal{M}}=\Omega \cap \mathcal{M}=\left\{\Omega_{i \mid \mathcal{M}}\right\}_{96}$ Each triangle in the input surface is split and assigned, to the incident Voronoi cells. Fig. 2 shows an example of RVD. The dual of the RVD is the primal trian $\overline{9}$ gulation, called the restricted Delaunay triangulationo (RDT). The RDT is the actual output of the remeshing framework.

\subsection{Feature-sensitive CVT extension}

Feature sensitive CVT is proposed by [10] to injeets normal anisotropy into the original CVT energy function as follows:

$$
F_{C V T_{f s}}(\mathbf{X})=\sum_{i=1}^{n} \sum_{f \in \Omega_{i \mid \mathcal{M}}} \int_{f} \rho(\mathbf{x})\left\|\mathbf{A}_{s}\left(\mathbf{N}^{f}\right)\left(\mathbf{x}-\mathbf{x}_{i}\right)\right\|^{2} \mathrm{~d} \sigma,
$$

where $\mathbf{N}^{f}$ is the normal of a triangle $f$ used for integration (see Fig. 2) and $\mathbf{A}_{s}(\mathbf{N})=(s-1) \mathbf{N} \mathbf{N}^{t}+\mathbf{I}_{3 \times 3}$. The parameter $s \geq 1$ controls the weight of the normal anisotropy. We set $s=5$ in all our experimental resules 6 as suggested in [10].

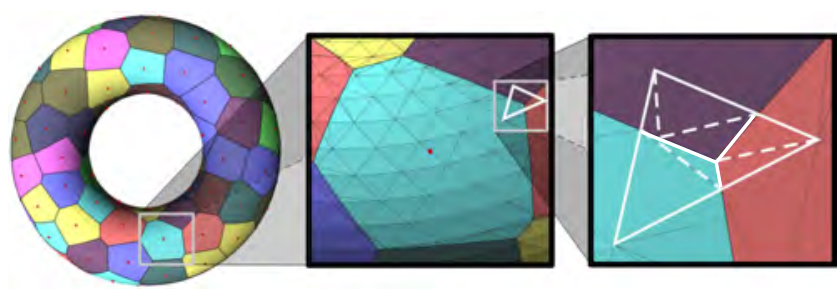

Fig. 2. Illustration of the RVD computation. All input triangles are assigned to their incident Voronoi cell(s). Some triangles are split in the process (middle). Splitting such a triangle produces multiple smaller triangles used for computing the integral of the energy function (dashed white triangles in the right).

\subsection{L-BFGS optimization}

The straightforward CVT implementation using Lloyd iterations moves vertices to the centroids of their corresponding Voronoi cells. This is a gradient decent algorithm with linear convergence [13]. Liu et al. [25] prove that the energy term has $C^{2}$ smoothness and can therefore be optimized using a quasi-Newton-like solver, such as L-BFGS [45]. Since the new introduced primal penalty term in Eqn. 3 is also quadratic, our objective function can be minimized efficiently by quasi-Newton solvers.

\section{Methodology}

In this section we first describe our novel penalty term and give further implementation details how the penalty term is embedded into the remeshing framework.

\subsection{Penalty term}

As discussed in the previous section, the minimization of the CVT energy is not sufficient to remove obtuse triangles, especially when using non-uniform density functions. By analyzing the configurations of CVT, we can observe that the longest edge of an obtuse triangle is always dual to a short Voronoi edge. An example is shown in Fig. 3. Inspired by this simple observation, we propose to add a penalty term in the CVT energy function for the primal triangulation RDT, that penalizes the short Voronoi edges. The modified CVT energy function for eliminating obtuse triangles is defined as follows:

$$
F(\mathbf{X})=F_{C V T_{f s}}(\mathbf{X})+\lambda R(\mathbf{X}),
$$

where $R(\mathbf{X})$ is the penalty term designed for preventing short Voronoi edges. It is defined as:

$$
R(\mathbf{X})=\sum_{i=1}^{n}\left\|\sum_{\left(\mathbf{x}_{i}, \mathbf{x}_{j}\right) \in R D T} w_{i, j}\left(\mathbf{x}_{i}-\mathbf{x}_{j}\right)\right\|^{2},
$$

where $w_{i, j}=\frac{\left\|\left(\mathbf{x}_{i}, \mathbf{x}_{j}\right)\right\|}{\| \text { dual }\left(\mathbf{x}_{i}, \mathbf{x}_{j}\right) \|+\epsilon}$. Here, a small value $\epsilon$ is used to avoid the denominator becoming zero. Also, 


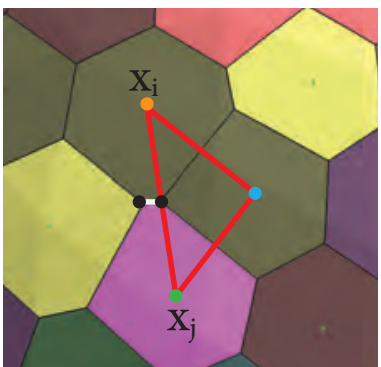

231

232

233

234

235

236

237

238

239

Fig. 3. Illustration of the relationship between shokto Voronoi edge and obtuse triangle. The obtuse triangl is shown in red, and the Voronoi edge that is dual tor the longest edge is shown in white.

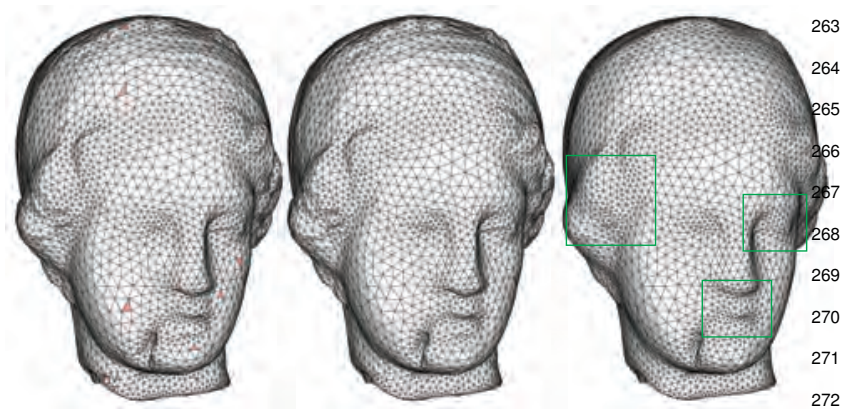

Fig. 4. Illustration of the effectiveness of the weight $\lambda^{273}$. Left: $\lambda=0$ is identical to CVT; middle: an appropriate value of $\lambda=1$ eliminates obtuse triangles while approximating the input mesh well; right: a larger value of $\lambda=10$ smoothes out the details.
CVT. This can also be observed in our results described in Sec. 5. For the RVD computation, we use the latest improved version presented in [28], which is able to handle surfaces containing thin sheets and self-intersections. There are four main steps of our framework.

Initial sampling. In this step, we randomly generate $n$ initial points on the mesh surface, with respect to a given density function $\rho(\mathbf{x})$ defined on the mesh vertices and piecewise-linearly interpolated over the mesh triangles. For non-uniform remeshing we use the local feature size (lfs) [47], which is a popular choice in the literature.

CVT optimization. We optimize the initial point set with the CVT optimizer without the penalty term and the feature sensitive constraint, typically for 30-50 iterations.

Valence optimization. The CVT energy function is nonconvex and has many local minima. Empirally, we can observe that vertices with valences smaller than 5 or larger than 7 , which are called bad-valence vertices in this paper, almost always correpsond to undesirable local minima. It has been proven that the global minimum of the CVT energy function is a regular hexagonal pattern [48] in the plane. While no similar proof exists for surfaces, other authors also observed that removing bad-valence vertices by perturbation reduces the CVT energy [49]. We solve this problem by perturbing the bad valence vertices locally. For each bad valence vertex, we randomly sample a new vertex inside the triangles in the onering neighborhood. The newly sampled vertex is projected onto the input mesh, and is inserted into the Delaunay triangulation. The old bad valence vertex is removed. Then Lloyd optimization is performed locally around these vertices (e.g., 3-ring neighborhood), while the others are fixed. Fig. 5 illustrates this process. This simple method works well since neither high valences $(>7)$ nor low valences $(<5)$ are not stable configurations. In all our tests, this simple perturbation can remove the bad-valence vertices without adding/removing/teleporting other vertices, which helps the global convergence of the optimization.
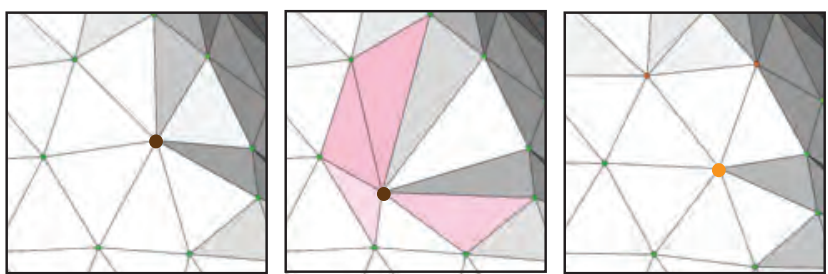

Fig. 5. Localized random optimization of bad-valence vertices. Green: valence 6; Orange: valence 7 and Blue: valence 5 . The dark color stands for high/low valences. Left: current configuration. Middle: a valence 8 vertex is perturbed. Right: after 3 steps of local Lloyd optimization, the high valence vertex is removed. 
Obtuse triangle removal. Once we have a well disos tributed point set, we add the penalty term in theo CVT energy function and start the second stage of $f_{1}$ the optimization. It removes the obtuse triangles whilez keeping the good global distribution of the verticest3 Finally, we optimize points again with the feature sen +4 sitive CVT (Eq. 3) and the penalty term for improving 5 the approximation quality.

\section{EXPERIMENTAL RESULtS}

In this section, we first analyze the remeshing qualzo ity of the state-of-the-art CVT framework. Next, wsen evaluate our algorithm and compare our method witha other remeshing techniques. The proposed approachas is implemented in $\mathrm{C}++$. The results shown in this 4 section are generated on an Intel X5680 Dual-Cores 3.33GHz CPU with 4GB memory and a 64-bit Winz6 dows 7 operating system.

Problem analysis. We analyze the remeshing qualityz8 of the proposed state-of-the-art remeshing frameworks with and without the feature sensitive extension. $W_{8}$ observed that the feature sensitive extension always has significantly more obtuse triangles, so we only show the results without the feature sensitive exten $\overline{3}_{33}$ sion here. We let the optimizer run until convergences Fig. 6 shows the number of obtuse triangles againşక the number of iterations. We can see that CVT cannot eliminate obtuse triangles even after hundreds of iterations, especially for non-uniform remeshing.

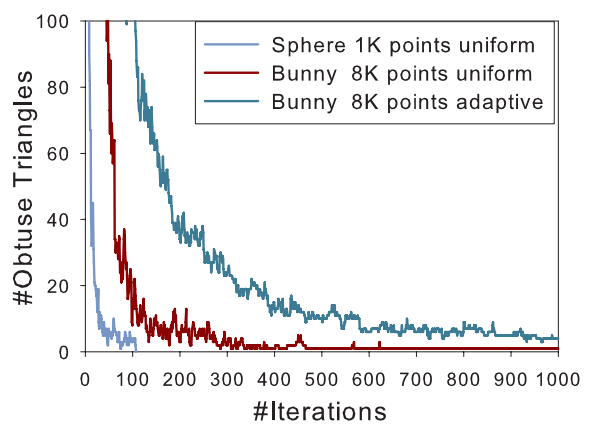

Fig. 6. The number of obtuse triangles against the number of iterations for CVT optimization. We can see that CVT is able to get rid of obtuse triangles in $\overbrace{3}$ smooth domain with a small number of points, e.g.g the sphere with $1 \mathrm{~K}$ points. However, if we increases the complexity of the domain, as well as the number of points, CVT is not able to generate non-obtusest remeshing even with thousands of iterations. ate Centroidal Voronoi Diagram (ACVD) [20], pacity Constrained CVT (CVT $\mathrm{Cap}_{\text {) }}$ [29], Maximal Poissondisk Sampling (MPS) [30] and Farthest Point Optimization (FPO) [31]. In the first comparison we chooses two example models for both uniform remeshing and adaptive remeshing, respectively. The comparison is summarized in Table 1. We can see that our algorithm is almost as good as $\mathrm{CVT}_{f s}$ in approximating the original mesh, while having significantly better angle quality than any other method assuming that both small and large angles are considered undesirable. In the second comparison we only compare with CVT and $\mathrm{CVT}_{f s}$ (See Table 2). Selected meshing results are visualized in Fig. 7 and more results are shown in the additional materials.

Parameter analysis. There are two main parameters used in our approach. One is the coefficient $\lambda$ of the penalty term (Eq. 2) and the other is the parameter $s$ that controls the scale of feature sensitive CVT. Assume that in the asymptotical setting, each restricted Voronoi cell becomes a regular hexagon, and the density function is a constant inside each cell. Denote $r$ as the edge length of a Voronoi edge, then the CVT energy is proportional to $r^{4}$, while the penalty term is proportional to $r^{2}$. Since $r^{2} \propto \frac{|M|}{n}(|M|$ is the area of the mesh surface), we multiply the penalty term by $\frac{|M|}{n}$ for normalization.

Another important parameter is the feature sensitive parameter $s$ in Eq. 2. Fig. 8 shows the influence of this parameter. In this example, we set $\lambda=1$ and increase the value of $s$. As shown in the figure, larger $s$ will introduce more bad-shaped triangles, while having smaller approximation errors, i.e., smaller Hausdorff distances.

Sharp feature handling. We provide an alternative way to handle the sharp features of CAD models or boundaries. We assume that the sharp features are tagged by the user or given as input. We restrict the vertices whose restricted Voronoi cells intersect with feature curves strictly on the feature, similar to previous approaches [24], [30]. The vertices of the input mesh that are incident to (more than) three feature edges are identified are as corners and will be sampled and kept unchanged during the optimization process. Fig. 9 shows an example of boundary handling of curved surfaces, and Fig. 11 shows another example of handling CAD models with structured feature skeletons.

Comparison. We first compare our results with the decimation-based non-obtuse remeshing [43] in Table 3. Our results always have minimal angles larger than $30^{\circ}$ while the results of [43] do not have this property. Further, our approximation quality is better.

\begin{tabular}{|c|c|c|c|c|c|c|}
\hline Model & Method & $|\mathbf{X}|$ & $|\triangle|$ & $\theta_{\min }$ & $\theta_{<30^{\circ}} \%$ & $d_{H}\left(\times 10^{-2}\right)$ \\
\hline \multirow{2}{*}{ Horse } & {$[43]$} & 9944 & 19880 & 15.03 & 0.18 & 1.31 \\
& Ours & 9944 & 19884 & $\mathbf{3 8 . 4}$ & $\mathbf{0}$ & $\mathbf{0 . 4 6}$ \\
\hline
\end{tabular}

TABLE 3

A comparison with the non-obtuse decimation [43]. 

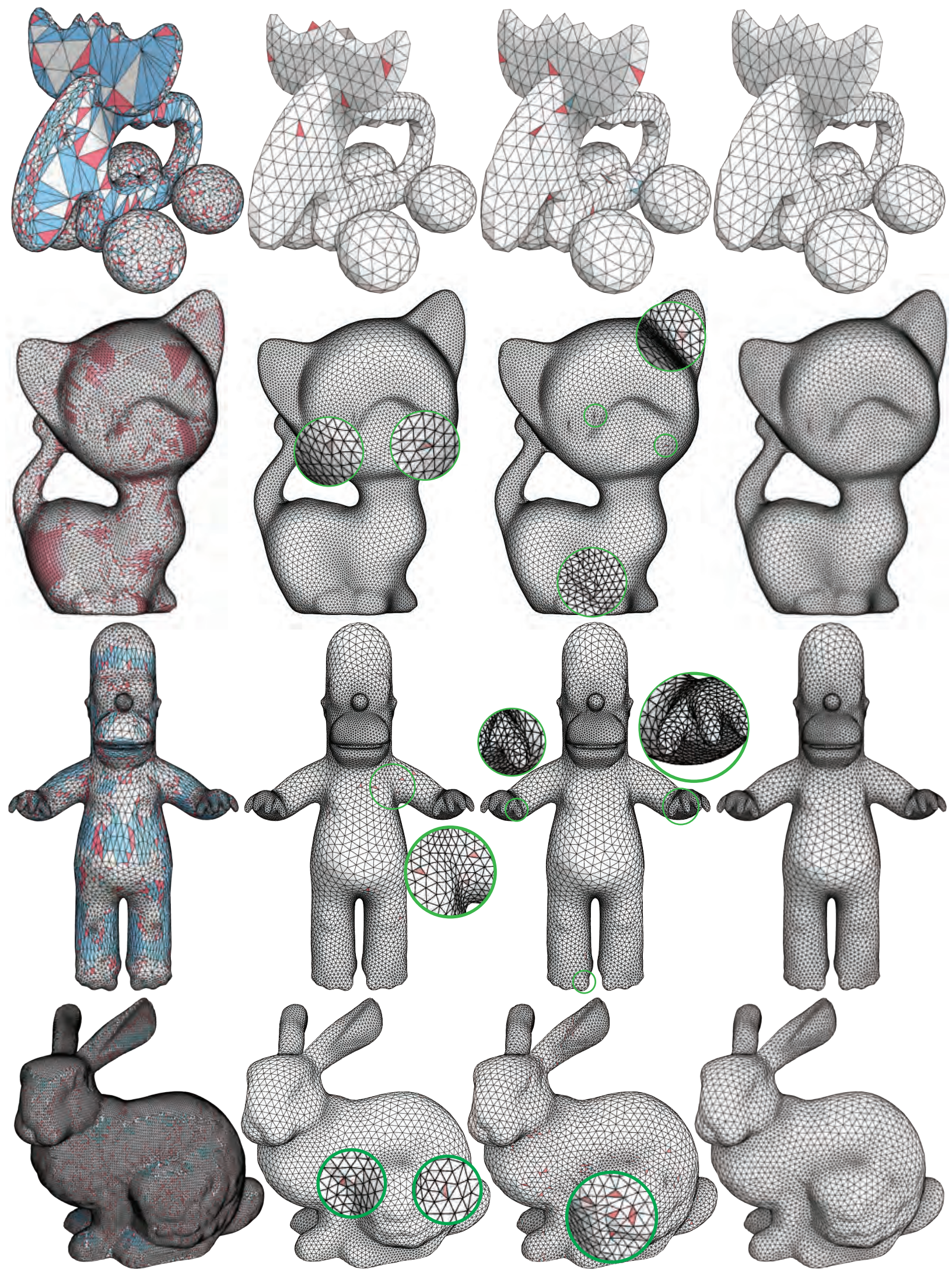

Fig. 7. Comparison of remeshing with CVT, $\mathrm{CVT}_{f s}$ and our method: uniform remeshing of the Elk model ( $1 \mathrm{~K}$ vertices), and adaptive remeshing of Kitten (9.8K vertices), Homer (7.5K vertices), and Bunny (8K vertices). The obtuse triangles are shown in pink, and triangles with $\theta_{\min } \leq 30^{\circ}$ are shown in blue. From left to right: input meshes, results of $\mathrm{CVT}_{1} \mathrm{CVT}_{f_{s}}$ and $C V T_{n o b}$ (ours). 


\begin{tabular}{|c|c|c|c|c|c|c|c|c|c|c|c|c|c|c|}
\hline Model & Method & $|\mathbf{X}|$ & $|\triangle|$ & $\triangle_{o b t}$ & $Q_{\min }$ & $Q_{a v g}$ & $\theta_{\min }$ & $\bar{\theta}_{\text {min }}$ & $\theta_{\max }$ & $\theta_{<30^{\circ}} \%$ & $\theta>90^{\circ} \%$ & $V_{567} \%$ & $d_{R M S}\left(\times 10^{-3}\right)$ & $d_{H}\left(\times 10^{-2}\right)$ \\
\hline \multirow{7}{*}{ Venus } & ACVD & $3.0 \mathrm{~K}$ & $6.0 \mathrm{~K}$ & 466 & 0.25 & 0.84 & 14.4 & 47.6 & 146.3 & 0.36 & 7.77 & 98.4 & 0.76 & 0.74 \\
\hline & MPS & $3.0 \mathrm{~K}$ & $6.0 \mathrm{~K}$ & 0 & 0.67 & 0.85 & 32.7 & 48.6 & 90.0 & 0 & 0 & 100 & 0.67 & 0.68 \\
\hline & FPO & $3.0 \mathrm{~K}$ & $6.0 \mathrm{~K}$ & 377 & 0.57 & 0.85 & 34.2 & 50.8 & 107.1 & 0 & 6.29 & 99.7 & 0.64 & 0.71 \\
\hline & $\mathrm{CVT}_{\text {cap }}$ & $3.0 \mathrm{~K}$ & $6.0 \mathrm{~K}$ & $1.0 \mathrm{~K}$ & 0.39 & 0.78 & 20.5 & 43.3 & 128.9 & 4.41 & 17.7 & 98.8 & 0.68 & 0.61 \\
\hline & CVT & $3.0 \mathrm{~K}$ & $6.0 \mathrm{~K}$ & 15 & 0.65 & 0.93 & 39.5 & 54.5 & 97.3 & 0 & 0.25 & 100 & 0.76 & 0.59 \\
\hline & $\mathrm{CVT}_{f s}$ & $3.0 \mathrm{~K}$ & $6.0 \mathrm{~K}$ & 49 & 0.61 & 0.91 & 27.2 & 53.0 & 102.7 & 0.03 & 0.82 & 99.8 & 0.32 & 0.31 \\
\hline & $\mathrm{CVT}_{n o b}$ & $3.0 \mathrm{~K}$ & $6.0 \mathrm{~K}$ & 0 & 0.73 & 0.96 & 37.4 & 55.9 & 86.1 & 0 & 0 & 100 & 0.62 & 0.56 \\
\hline \multirow{7}{*}{ Genus } & ACVD & $6.5 \mathrm{~K}$ & $13 \mathrm{~K}$ & 913 & 0.47 & 0.85 & 29.0 & 48.1 & 118.6 & 0.06 & 7.02 & 98.1 & 0.45 & 0.44 \\
\hline & MPS & $6.5 \mathrm{~K}$ & $13 \mathrm{~K}$ & 0 & 0.66 & 0.85 & 31.3 & 48.5 & 90.0 & 0 & 0 & 100 & 0.41 & 0.65 \\
\hline & FPO & $6.5 \mathrm{~K}$ & $13 \mathrm{~K}$ & 737 & 0.55 & 0.86 & 33.1 & 50.9 & 109.3 & 0 & 6.14 & 99.6 & 0.42 & 0.59 \\
\hline & $\mathrm{CVT}_{\text {cap }}$ & $6.5 \mathrm{~K}$ & $13 \mathrm{~K}$ & $2.3 \mathrm{~K}$ & 0.38 & 0.78 & 16.8 & 42.6 & 130.0 & 5.91 & 18.3 & 98.6 & 0.44 & 0.45 \\
\hline & CVT & $6.5 \mathrm{~K}$ & $13 \mathrm{~K}$ & 27 & 0.66 & 0.94 & 38.7 & 54.6 & 96.8 & 0 & 0.21 & 100 & 0.47 & 0.52 \\
\hline & $\mathrm{CVT}_{f_{s}}$ & $6.5 \mathrm{~K}$ & $13 \mathrm{~K}$ & 50 & 0.62 & 0.93 & 34.1 & 54.5 & 100.3 & 0 & 0.38 & 100 & 0.21 & 0.22 \\
\hline & $\mathrm{CVT}_{n o b}$ & $6.5 \mathrm{~K}$ & $13 \mathrm{~K}$ & 0 & 0.72 & 0.95 & 36.0 & 55.6 & 88.9 & 0 & $\mathbf{0}$ & 100 & 0.23 & 0.31 \\
\hline \multirow{7}{*}{ Homer } & ACVD & $7.5 \mathrm{~K}$ & $15 \mathrm{~K}$ & $2.7 \mathrm{~K}$ & 0.04 & 0.80 & 2.11 & 44.4 & 174.1 & 6.18 & 17.7 & 94.4 & 0.69 & 0.33 \\
\hline & MPS & $7.5 \mathrm{~K}$ & $15 \mathrm{~K}$ & $1.6 \mathrm{~K}$ & 0.56 & 0.82 & 30.1 & 46.4 & 105.8 & 0 & 10.8 & 100 & 0.52 & 0.30 \\
\hline & $\mathrm{FPO}$ & $7.5 \mathrm{~K}$ & $15 \mathrm{~K}$ & $1.2 \mathrm{~K}$ & 0.50 & 0.85 & 28.2 & 49.9 & 116.0 & 0.01 & 7.52 & 98.9 & 0.43 & 0.30 \\
\hline & $\mathrm{CVT}_{\text {cap }}$ & $7,5 \mathrm{~K}$ & $15 \mathrm{~K}$ & $1.6 \mathrm{~K}$ & 0.37 & 0.83 & 21.3 & 46.2 & 131.1 & 0.98 & 10.66 & 95.8 & 0.37 & 0.20 \\
\hline & CVT & $7.5 \mathrm{~K}$ & $15 \mathrm{~K}$ & 56 & 0.65 & 0.93 & 38.1 & 54.0 & 98.2 & 0 & 0.37 & 100 & 0.49 & 0.20 \\
\hline & $\mathrm{CVT}_{f s}$ & $7.5 \mathrm{~K}$ & $15 \mathrm{~K}$ & 108 & 0.50 & 0.91 & 27.4 & 52.5 & 113.1 & 0.01 & 7.21 & 99.9 & 0.24 & 0.16 \\
\hline & $\mathrm{CVT}_{n o b}$ & $7.5 \mathrm{~K}$ & $15 \mathrm{~K}$ & 0 & 0.69 & 0.94 & 33.7 & 54.5 & 85.4 & 0 & 0 & 100 & 0.31 & 0.17 \\
\hline \multirow{7}{*}{ Bunny } & ACVD & $8.0 \mathrm{~K}$ & $16 \mathrm{~K}$ & $3.3 \mathrm{~K}$ & 0.10 & 0.77 & 4.76 & 42.4 & 165.9 & 9.96 & 20.5 & 92.2 & 0.61 & 0.59 \\
\hline & MPS & $8.3 \mathrm{~K}$ & $16.6 \mathrm{~K}$ & $1.3 \mathrm{~K}$ & 0.400 & 0.83 & 33.9 & 53.9 & 103.0 & 0 & 0.29 & 99.8 & 0.47 & 0.34 \\
\hline & FPO & $8.0 \mathrm{~K}$ & $16 \mathrm{~K}$ & $1.6 \mathrm{~K}$ & 0.39 & 0.84 & 22.6 & 48.7 & 128.5 & 0.56 & 0.29 & 98.0 & 0.45 & 0.34 \\
\hline & $\mathrm{CVT}_{\text {cap }}$ & $8.0 \mathrm{~K}$ & $16 \mathrm{~K}$ & $1.4 \mathrm{~K}$ & 0.39 & 0.84 & 15.6 & 47.3 & 125.1 & 1.04 & 8.46 & 98.7 & 0.43 & 0.24 \\
\hline & CVT & $8.0 \mathrm{~K}$ & $16 \mathrm{~K}$ & 10 & 0.64 & 0.93 & 34.8 & 54.2 & 98.8 & 0 & 0.06 & 99.9 & 0.53 & 0.37 \\
\hline & $\mathrm{CVT}_{f s}$ & $8.0 \mathrm{~K}$ & $16 \mathrm{~K}$ & 492 & 0.32 & 0.89 & 12.5 & 50.6 & 128.8 & 0.43 & 3.06 & 99.5 & 0.43 & 0.26 \\
\hline & $\mathrm{CVT}_{n o b}$ & $8.0 \mathrm{~K}$ & $16 \mathrm{~K}$ & 0 & 0.75 & 0.94 & 35.5 & 55.0 & \begin{tabular}{|l|}
84.2 \\
\end{tabular} & 0 & 0 & 100 & 0.44 & 0.49 \\
\hline
\end{tabular}

TABLE 1

Comparison of remeshing quality with previous techniques. The best result of each measurement is marked in bold font. $|\mathbf{X}|$ is the number of vertices; $|\triangle|$ is the number of triangles; $\left|\triangle_{\text {obt }}\right|$ is the number of obtuse triangles; $Q_{\text {min }}$ is the minimal triangle quality, where the quality of a triangle is $Q(\mathbf{t})=\frac{6}{\sqrt{3}} \frac{S_{\mathbf{t}}}{p_{\mathbf{t}} h_{\mathbf{t}}}$, where $S_{\mathbf{t}}$ is the area of $\mathbf{t}, p_{\mathbf{t}}$ is the half-perimeter of $\mathbf{t}$ and $h_{\mathbf{t}}$ the the longest edge length of $\mathbf{t}$ [50]; $Q_{\text {avg }}$ is the average of the triangle qualities;

$\theta_{\min }$ is the minimal angle; $\bar{\theta}_{\min }$ is the average of minimal angles of each triangle; $\theta_{\max }$ is the maximal angle,

$\theta_{<30^{\circ}} \%$ is the percentage of triangles with angles smaller than $30^{\circ} ; \theta_{>90^{\circ}} \%$ is the percentage of obtuse triangles;

$V_{567} \%$ is the percentage of the valence 5,6 , and 7 vertices; $d_{R M S}$ is the root mean square distance, and $d_{H}$ is the Hausdorff distance between the remesh and the input surface, which is measured by Metro [51].

\begin{tabular}{|c|c|c|c|c|c|c|c|c|c|c|c|c|c|c|}
\hline Model & Method & $\mid \mathbf{X}$ & $|\triangle|$ & $\triangle_{\text {obt }}$ & $Q_{\min }$ & $Q_{\text {avg }}$ & $\theta_{\min }$ & $\bar{\theta}_{\min }$ & $\theta_{\max }$ & $\theta_{<30^{\circ} \%}$ & $\theta_{>90^{\circ}} \%$ & $V_{567} \%$ & $d_{R M S}\left(\times 10^{-3}\right)$ & $d_{H}\left(\times 10^{-2}\right)$ \\
\hline \multirow{3}{*}{ Elk } & $\mathrm{CVT}$ & $1.0 \mathrm{~K}$ & $2.0 \mathrm{~K}$ & 8 & 0.66 & 0.92 & $\mathbf{3 6 . 9}$ & 53.7 & 95.0 & $\mathbf{0}$ & 0.40 & 97.3 & 3.64 & 1.48 \\
& $\mathrm{CVT}_{f s}$ & $1.0 \mathrm{~K}$ & $2.0 \mathrm{~K}$ & 29 & 0.50 & 0.90 & 22.6 & 51.6 & 104.0 & 0.70 & 1.45 & 97.5 & $\mathbf{1 . 5 8}$ & $\mathbf{0 . 7 5}$ \\
& $\mathrm{CVT}_{\text {nob }}$ & $1.0 \mathrm{~K}$ & $2.0 \mathrm{~K}$ & $\mathbf{0}$ & 0.70 & $\mathbf{0 . 9 3}$ & 36.2 & $\mathbf{5 3 . 9}$ & $\mathbf{8 8 . 2}$ & $\mathbf{0}$ & $\mathbf{0}$ & $\mathbf{9 7 . 8}$ & 1.93 & 1.14 \\
\hline \multirow{3}{*}{ Botijo } & $\mathrm{CVT}$ & $5.0 \mathrm{~K}$ & $10 \mathrm{~K}$ & 8 & 0.67 & 0.93 & $\mathbf{3 8 . 2}$ & $\mathbf{5 4 . 2}$ & 94.9 & $\mathbf{0}$ & 0.08 & 100 & 0.74 & 0.33 \\
& $\mathrm{CVT}_{f S}$ & $5.0 \mathrm{~K}$ & $10 \mathrm{~K}$ & 231 & 0.51 & 0.89 & 26.5 & 51.1 & 114.8 & 0.06 & 2.31 & 99.7 & $\mathbf{0 . 3 6}$ & 0.33 \\
& $\mathrm{CVT}_{\text {nob }}$ & $5.0 \mathrm{~K}$ & $10 \mathrm{~K}$ & $\mathbf{0}$ & $\mathbf{0 . 7 0}$ & $\mathbf{0 . 9 4}$ & 31.1 & $\mathbf{5 4 . 2}$ & $\mathbf{8 8 . 4}$ & $\mathbf{0}$ & $\mathbf{0}$ & $\mathbf{1 0 0}$ & 0.48 & $\mathbf{0 . 3 2}$ \\
\hline \multirow{3}{*}{ Rockerarm } & $\mathrm{CVT}^{*}$ & $5.8 \mathrm{~K}$ & $11.6 \mathrm{~K}$ & 5 & 0.67 & $\mathbf{0 . 9 4}$ & $\mathbf{3 7 . 9}$ & 54.7 & 94.7 & $\mathbf{0}$ & 0.04 & 100 & 0.79 & 0.46 \\
& $\mathrm{CVT}_{f s}$ & $5.8 \mathrm{~K}$ & $11.6 \mathrm{~K}$ & 391 & 0.28 & 0.88 & 10.5 & 50.5 & 117.5 & 0.49 & 3.37 & 99.3 & $\mathbf{0 . 2 8}$ & $\mathbf{0 . 3 1}$ \\
& $\mathrm{CVT}_{\text {nob }}$ & $5.8 \mathrm{~K}$ & $11.6 \mathrm{~K}$ & $\mathbf{0}$ & $\mathbf{0 . 7 4}$ & $\mathbf{0 . 9 4}$ & 31.7 & $\mathbf{5 4 . 9}$ & $\mathbf{8 9 . 9}$ & $\mathbf{0}$ & $\mathbf{0}$ & $\mathbf{1 0 0}$ & 0.53 & 0.36 \\
\hline \multirow{3}{*}{ Kitten } & $\mathrm{CVT}^{*}$ & $9.8 \mathrm{~K}$ & $19.6 \mathrm{~K}$ & 6 & 0.67 & 0.94 & $\mathbf{3 9 . 2}$ & 54.8 & 95.6 & $\mathbf{0}$ & 0.03 & 99.9 & 0.32 & 0.22 \\
& $\mathrm{CVT}_{f s}$ & $9.8 \mathrm{~K}$ & $19.6 \mathrm{~K}$ & 119 & 0.52 & 0.93 & 26.4 & 53.9 & 112.6 & 0.03 & 0.61 & 99.9 & $\mathbf{0 . 1 7}$ & $\mathbf{0 . 1 4}$ \\
& $\mathrm{CVT}_{\text {nob }}$ & $9.8 \mathrm{~K}$ & $19.6 \mathrm{~K}$ & $\mathbf{0}$ & $\mathbf{0 . 7 1}$ & $\mathbf{0 . 9 5}$ & 33.6 & $\mathbf{5 5 . 3}$ & $\mathbf{8 7 . 1}$ & $\mathbf{0}$ & $\mathbf{0}$ & $\mathbf{1 0 0}$ & 0.23 & 0.21 \\
\hline
\end{tabular}

\section{TABLE 2}

Comparison of remeshing quality with other CVT-based approaches.

optimization for non-obtuse triangles, the method casa be configured to eliminate non-obtuse triangles byø3 random resampling. We can observe that MPS is abbet to generate non-obtuse meshes for uniform remeshing problems. However, it never converges for adaptive remeshing. 


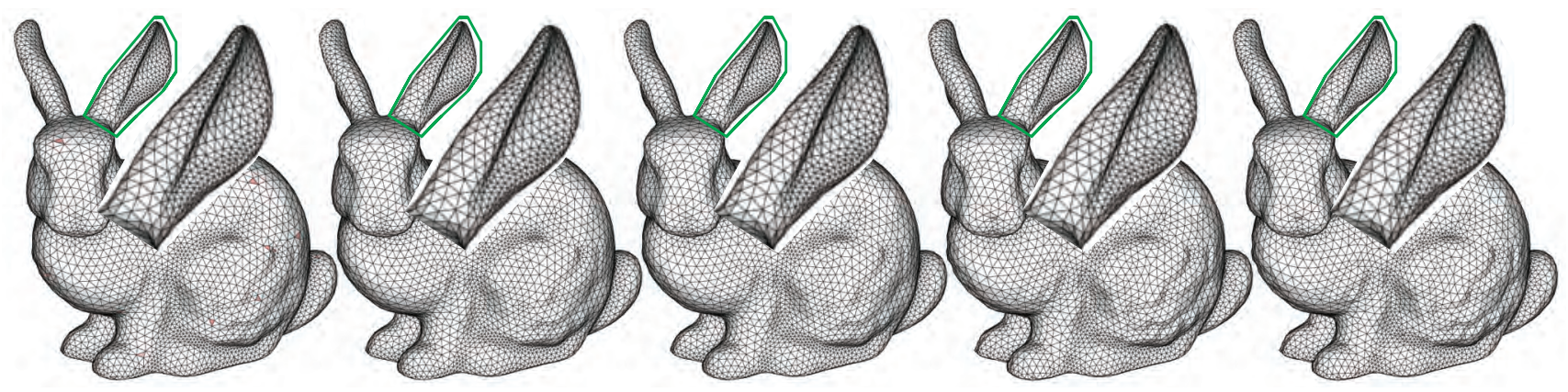

Fig. 8. Illustration of the remeshing results with different parameters for feature sensitive CVT. (a) result of CVT: $s=1, \lambda=0,\left|\triangle_{o b t}\right|=79, d_{H}=0.41 \%$; (b) result with CVT + penalty term: $s=1, \lambda=1,\left|\triangle_{o b t}\right|=0, d_{H}=0.36 \%$, (c, d, e) result of $\mathrm{CVT}_{f s}+$ penalty term: $s=5, \lambda=1,\left|\triangle_{\text {obt }}\right|=0, d_{H}=0.33 \% ; s=8, \lambda=1,\left|\triangle_{o b t}\right|=113, d_{H}=$ $0.20 \% ; s=10, \lambda=1,\left|\triangle_{o b t}\right|=210, d_{H}=0.18 \%$.
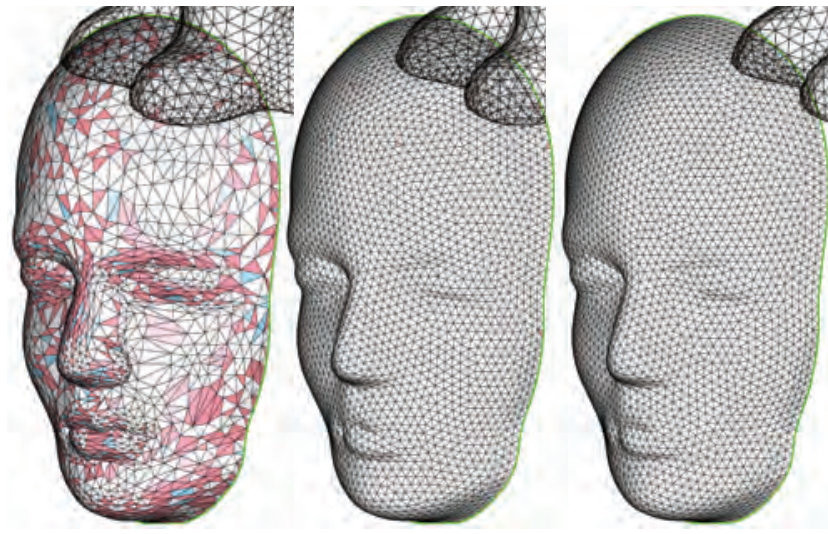

Fig. 9. Boundary and feature handling. Left: input mesh (the boundary curve is shown in green); middle: result of CVT with 14 obtuse triangles; right: our nonobtuse remeshing.
Finally we compare with the recent work using weighted triangulations that aims at generating well-centered meshes [4], which is an improvement over the primal/dual Hodge-Optimized Triang i $^{87}$ lation (HOT) [37]. As shown in Fig. 10, although the $e^{38}$ weighted triangulation approach creates meshes with ${ }^{9}$ elegant primal/dual structure, it is not able to eliminate all the obtuse triangles. Since the weighted cente $e^{391}$ of each triangle is not the circumcenter anymore,r, in the context of weighted triangulations, the wel193 centered mesh is typically not a non-obtuse mesh. ${ }^{394}$ Limitations. Our algorithm might not be successful in the presence of noise or when the density function changes dramatically (see Fig. 11 (top)). Our algorithm cannot eliminate all obtuse triangles along the sharp features. One example is the uniform remeshings of the Joint model as shown in Fig. 11(bottom left3ag This model contains sharp features and planar regionso on each side of the features. In this case, once then valence of a vertex on a sharp feature is less thana six, there will be an obtuse angle on the side whenes the vertex has only one neighbor. Another failures

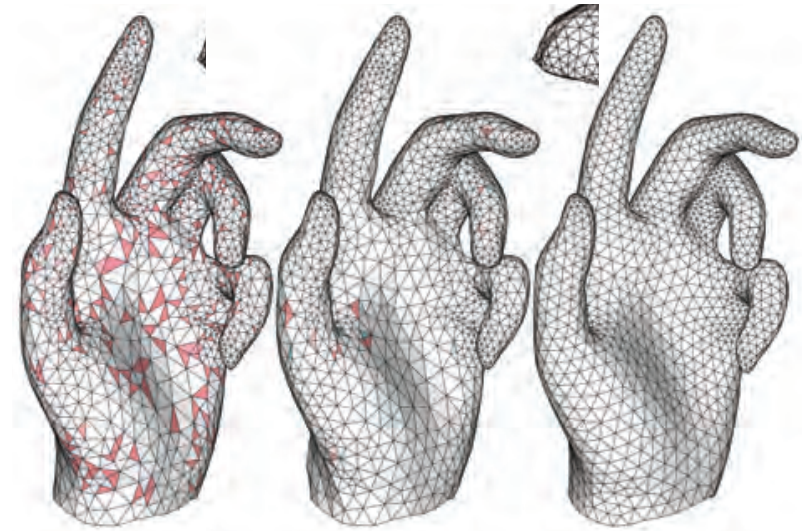

Fig. 10. Comparison with well-centered meshes generated by weighted triangulation [4]. The same number of vertices are used in the remeshing. Left: input mesh with 687 obtuse triangles (16.0\%); middle: result of the weighted triangulation [4] with 17 triangles with small angles $\left(<30^{\circ}\right)$ and 93 obtuse triangles $(2.17 \%)$; right: result of our non-obtuse remeshing.

example for adaptive remeshing of the Sculpt models is shown in the bottom right of Fig. 11. Some of these problems could be improved in future work, but certain configurations of sharp features inherently lead to small undesirable angles (e.g., two features curves joining with a small angle). Another limitation is that we do not provide a theoretical proof of the convergence analysis of the proposed primal penalty term that could guarantee the $\mathrm{max} / \mathrm{min}$ angle bounds of the resulting meshes. We would like to address above problems as future works.

\section{Conclusion ANd Future Work}

We present a novel remeshing algorithm that reduces the number of triangles with small angles (less than 30 degrees) and triangles with large (obtuse) angles. We augment the original CVT formulation by a primal penalty term preventing short Voronoi edges. In this formulation, the dual term helps to avoid 

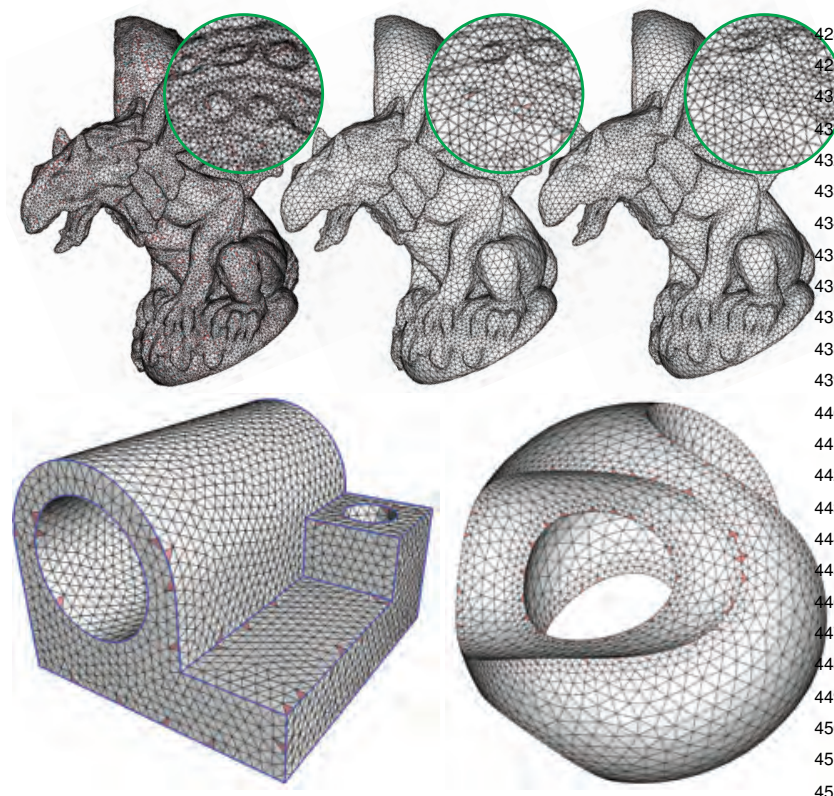

439

440

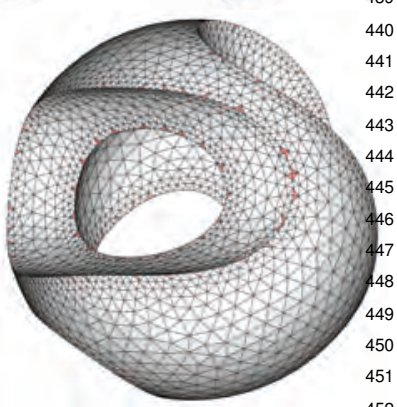

Fig. 11. Unsatisfied examples. Top row: our algorith $m_{454}^{453}$ fail to eliminate all the obtuse triangles of the Gargoyles model that has dramatically changed density functiont ${ }^{6}$ (left to right: input, result of CVT with 591 bad triangle ${ }_{458}^{457}$ and final result with 143 bad triangles). As shown in thes top right, an obtuse appears in the transitional regiot9 where the size of triangles changes quickly. Botto $44_{42}^{11}$ row: Uniform and adaptive remeshing of the Joint ands the Sculpt models with $4 \mathrm{~K}$ vertices, respectively. Ou\$4 algorithm cannot eliminate all the obtuse triangles thata are adjacent to sharp features. The angle bounds? are $\left[35.4^{\circ}, 105.0^{\circ}\right]$ and $\left[31.5^{\circ}, 101.1^{\circ}\right]$ for each modetos, respectively.

small angles and the primal term helps to avoid larges angles. In future work, we would like to look for ne $4_{475}^{474}$ applications of our non-obtuse remeshing techniqu 475 and extend our concept of primal/dual optimizationt to volumetric tetrahedral mesh generation, which ${ }_{479}^{478}$ preferred in applications such as fluid simulation. $\quad 480$

\section{ACKNOWLEDGEMENTS}

We would like to thank Zhonggui Chen, Fernando dees Goes and Sebastien Valette for providing the data and 46 executables, Bruno Lévy for sharing the code of feas7 ture sensitive CVT, and Lubin Fan for the rendering

\section{REFERENCES}

[1] M. Botsch, L. Kobbelt, M. Pauly, P. Alliez, and B. Lévy, Polygotqu Mesh Processing. AK Peters, 2010.

[2] Q. Du, D. Wang, and L. Zhu, "On mesh geometry and stiffness5 matrix conditioning for general finite element spaces," SIAA\&6 J. Numer. Anal., vol. 47, no. 2, pp. 1421-1444, 2009.

[3] K. Crane, F. de Goes, M. Desbrun, and P. Schröder, "Digitads geometry processing with discrete exterior calculus," in ACAd9 SIGGRAPH 2013 Courses, 2013, pp. 7:1-7:126.

500

[4] F. de Goes, P. Memari, P. Mullen, and M. Desbrun, "Weightesd triangulations for geometry processing," ACM Trans. on Grapkor ics, vol. 33, no. 3, pp. 28:1-28:13, 2014.
[5] P. Heckbert and M. Garland, "Survey of polygonal surface simplification algorithms," in SIGGRAPH 97 Course Notes: Multiresolution Surface Modeling, 1997.

[6] M. Botsch and L. Kobbelt, "A remeshing approach to multiresolution modeling," in Proc. of Symp. of Geometry Processing, 2004, pp. 189-196.

[7] J. Schreiner, C. E. Scheidegger, S. Fleishman, and C. T. Silva "Direct (re)meshing for efficient surface processing," Computer Graphics Forum (Proc. EUROGRAPHICS), vol. 25, no. 3, pp. 527-536, 2006.

[8] S.-W. Cheng, T. K. Dey, and J. R. Shewchuk, Delaunay Mesh Generation. CRC Press, 2012.

[9] P. Alliez, D. Cohen-Steiner, O. Devillers, B. Lévy, and M. Desbrun, "Anisotropic polygonal remeshing," ACM Trans. on Graphics (Proc. SIGGRAPH), vol. 22, no. 3, pp. 485-493, 2003.

[10] B. Lévy and Y. Liu, " $L_{p}$ centroidal Voronoi tesselation and its applications," ACM Trans. on Graphics (Proc. SIGGRAPH), vol. 29, no. 4, pp. 119:1-11, 2010.

[11] P. Alliez, G. Ucelli, C. Gotsman, and M. Attene, "Recent advances in remeshing of surfaces," in Shape Analysis and Structuring, 2008, pp. 53-82.

[12] A. Okabe, B. Boots, K. Sugihara, and S. N. Chiu, Spatial Tessellations: Concepts and Applications of Voronoi Diagrams, 2nd ed. Wiley, 2000.

[13] Q. Du, V. Faber, and M. Gunzburger, "Centroidal Voronoi tessellations: applications and algorithms," SIAM Review, vol. 41, pp. 637-676, 1999.

[14] P. Alliez, M. Meyer, and M. Desbrun, "Interactive Geometry Remeshing," ACM Trans. on Graphics (Proc. SIGGRAPH), vol. 21(3), pp. 347-354, 2002.

[15] P. Alliez, É. C. d. Verdiére, O. Devillers, and M. Isenburg, "Isotropic surface remeshing," in Shape Modeling International - SMI, 2003, pp. 49-58.

[16] V. Surazhsky, P. Alliez, and C. Gotsman, "Isotropic remeshing of surfaces: a local parameterization approach," in 12th Intl. Meshing Roundtable, 2003, pp. 204-231.

[17] M. Nieser, J. Palacios, K. Polthier, and E. Zhang, "Hexagonal global parameterization of arbitrary surfaces," IEEE Trans. on Vis. and Comp. Graphics, vol. 18, no. 6, pp. 865-878, 2011.

[18] S. A. Lloyd, "Least squares quantization in PCM," IEEE Transactions on Information Theory, vol. 28, no. 2, pp. 129-137, 1982.

[19] G. Rong, M. Jin, L. Shuai, and X. Guo, "Centroidal Voronoi tessellation in universal covering space of manifold surfaces," Comp. Aided Geom. Design, vol. 28, no. 8, pp. 475-496, 2011.

[20] S. Valette, J.-M. Chassery, and R. Prost, "Generic remeshing of 3D triangular meshes with metric-dependent discrete Voronoi diagrams," IEEE Trans. on Vis. and Comp. Graphics, vol. 14, no. 2, pp. 369-381, 2008.

[21] G. Peyré and L. D. Cohen, "Geodesic remeshing using front propagation," Int. J. Comput. Vision, vol. 69, no. 1, pp. 145-156, 2006.

[22] Y. Fu and B. Zhou, "Direct sampling on surfaces for high quality remeshing," in ACM symposium on Solid and physical modeling, 2008, pp. 115-124.

[23] X. Wang, X. Ying, Y.-J. Liu, S.-Q. Xin, W. Wang, X. Gu, W. Mueller-Wittig, and Y. He, "Intrinsic computation of centroidal Voronoi tessellation (CVT) on meshes," Computer-Aided Design, vol. 58, no. 0, pp. $51-61,2015$.

[24] D.-M. Yan, B. Lévy, Y. Liu, F. Sun, and W. Wang, "Isotropic remeshing with fast and exact computation of restricted Voronoi diagram," Computer Graphics Forum (Proc. SGP), vol. 28, no. 5, pp. 1445-1454, 2009.

[25] Y. Liu, W. Wang, B. Lévy, F. Sun, D.-M. Yan, L. Lu, and C. Yang, "On centroidal Voronoi tessellation - energy smoothness and fast computation," ACM Trans. on Graphics, vol. 28, no. 4, pp. 101:1-101:11, 2009.

[26] B. Lévy and N. Bonneel, "Variational anisotropic surface meshing with Voronoi parallel linear enumeration," in Proceedings of the 21st International Meshing Roundtable, 2012, pp. 349-366.

[27] B. Lévy, "Restricted voronoi diagrams for (re)-meshing surfaces and volumes," in Curves and Surfaces, 2014, code download: http://gforge.inria.fr/projects/geogram/.

[28] D.-M. Yan, G. Bao, X. Zhang, and P. Wonka, "Low-resolution remeshing using the localized restricted Voronoi diagram," IEEE Trans. on Vis. and Comp. Graphics, vol. 20, no. 10, pp. 418-1427, 2014. 
[29] Z. Chen, Z. Yuan, Y.-K. Choi, L. Liu, and W. Wang, "Variationads blue noise sampling," IEEE Trans. on Vis. and Comp. Graphicsia vol. 18, no. 10, pp. 1784-1796, 2012.

30] D.-M. Yan and P. Wonka, "Gap processing for adaptive maxize mal Poisson-disk sampling," ACM Trans. on Graphics, vol. 328,7 no. 5, pp. 148:1-148:15, 2013.

578

[31] D.-M. Yan, J. Guo, X. Jia, X. Zhang, and P. Wonka, "Blue79 noise remeshing with farthest point optimization," Computb80 Graphics Forum (Proc. SGP), vol. 33, no. 5, pp. 167-176, 2014581

[32] V. Nivoliers, D.-M. Yan, and B. Lévy, "Fitting polynomiad2 surfaces to triangular meshes with Voronoi squared distanse3 minimization," Eng. with Comput., vol. 30, no. 3, pp. 289-308вa 2014.

[33] H. Pan, Y.-K. Choi, Y. Liu, W. Hu, Q. Du, K. Polthier, C. Zhang, and W. Wang, "Robust modeling of constant mean curvature surfaces," ACM Trans. on Graphics (Proc. SIGGRAPH), vol. 31, no. 4 , p. 85,2012

[34] D. Sieger, P. Alliez, and M. Botsch, "Optimizing Voronoi diagrams for polygonal finite element computations," in Proceedings of the 19th International Meshing Roundtable, IMR 2010, 2010, pp. 335-350.

[35] E. Vanderzee, A. N. Hirani, D. Guoy, and E. Ramos, "Wellcentered triangulation," SIAM J. Sci. Comput., vol. 31, no. 6, p. 4497C4523, 2010

[36] F. Sun, Y.-K. Choi, W. Wang, D.-M. Yan, Y. Liu, and B. Lévy, "Obtuse triangle suppression in anisotropic meshes," Comp. Aided Geom. Design, vol. 28, no. 9, pp. 537-548, 2011.

[37] P. Mullen, P. Memari, F. de Goes, and M. Desbrun, "Hot: Hodge-optimized triangulations," ACM Trans. on Graphics (Proc. SIGGRAPH), vol. 30, no. 4, pp. 103:1-103:12, July 2011.

[38] J. R. Shewchuk, "What is a good linear element? interpolation, conditioning, and quality measures," in 11th Intl. Meshing Roundtable, 2002, pp. 115-126.

[39] B. S. Baker, E. Grosse, and C. S. Raffery, "Nonobtuse triangulation of polygons," Disc. \& Comp. Geom., vol. 3, pp. 147-168, 1988.

[40] M. Bern, S. Mitchell, and J. Ruppert, "Linear-size nonobtuse triangulation of polygons," in ACM Symp. on Comp. Geom., 1994, pp. 221-230.

[41] H. Erten and A. Üngör, “Computing acute and non-obtuse, triangulations," in CCCG 2007, 2007, pp. 205-208.

[42] H. Erten and A. Üngör, "Computing triangulations withouf small and large angles," in Sixth International Symposium on Voronoi Diagrams, ISVD 2009, 2009, pp. 192-201.

[43] J. Li and H. Zhang, "Nonobtuse remeshing and decimation in Proc. of Eurographics Symposium on Geometry Processing, 200659 p. Proc. of Symp. of Geometry Processing.

[44] H. Edelsbrunner and N. R. Shah, "Triangulating topological spaces," IJCGA, vol. 7, no. 4, pp. 365-378, 1997.

[45] D. C. Liu and J. Nocedal, "On the limited memory bfgs method for large scale optimization," Math. Program., vol. 45, no. 3, $\mathrm{pg}_{96}$ 503-528, 1989.

[46] R. J. Renka, "Two simple methods for improving a triangle mesh surface," Computer Graphics Forum, p. to appear, 2015.

[47] N. Amenta, M. Bern, and M. Kamvysselis, "A new Voronoibased surface reconstruction algorithm," in Proc. ACM SIGGRAPH, 1998, pp. 415-421.

[48] G. F. Tóth, "A stability criterion to the moment theorem," Studia Scientiarum Mathematicarum Hungarica, vol. 38, p. 209C224, 2001.

[49] L. Lu, F. Sun, H. Pan, and W. Wang, "Global optimization of centroidal voronoi tessellation with monte carlo approach," IEEE Trans. on Vis. and Comp. Graphics, vol. 18, no. 11, pp. 1880-1890, 2012.

[50] P. Frey and H. Borouchaki, "Surface mesh evaluation," in 6th Intl. Meshing Roundtable, 1997, pp. 363-374.

[51] P. Cignoni, C. Rocchini, and R. Scopigno, "Metro: measuring error on simplified surfaces," Computer Graphics Forum, vol. 17, no. 2, pp. 167-174, 1998.

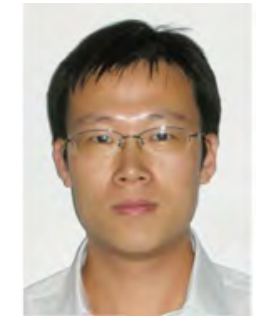

Dong-Ming Yan received the PhD degree from Hong Kong University in 2010, and his Bachelors and Masters degrees from Tsinghua University in 2002 and 2005, respectively. He is a research scientist at King Abdullah University of Science and Technology (KAUST) and an associate professor at the National Laboratory of Pattern Recognition of the Institute of Automation, Chinese Academy of Sciences. His research interests cessing and visualization

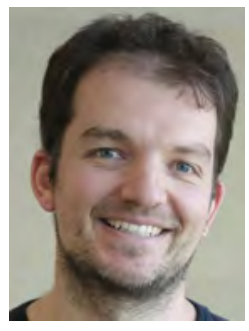

Peter Wonka received his PhD in computer science and his MS in urban planning from the Technical University of Vienna, Vienna, Austria, in 2001 and 2002, respectively. He was a postdoctoral researcher at the Georgia Institute of Technology, Atlanta, GA, for two years. He is currently a professor at the Computer, Electrical and Mathematical Science and Engineering Division, King Abdullah University of Science and Technology, Thuwal, Saudi Arabia, and also an associate professor at Arizona State University, Tempe, AZ, USA. His research interests include computer graphics, visualization, computer vision, remote sensing, image processing, and machine learning. 